\title{
ADAMTS13 Deficiency Worsens Colitis and Exogenous ADAMTS13 Administration Decreases Colitis Severity in Mice
}

\author{
Naamah L. Zitomersky ${ }^{1,2,3}$ Melanie Demers ${ }^{2,3}$ Kimberly Martinod ${ }^{2,3}$ Maureen Gallant ${ }^{3}$ \\ Stephen M. Cifuni ${ }^{3}$ Amlan Biswas ${ }^{1,2}$ Scott Snapper ${ }^{1,4,5}$ Denisa D. Wagner $2,3,6$
}

\footnotetext{
${ }^{1}$ Division of Gastroenterology, Hepatology, and Nutrition, Boston Children's Hospital, Boston, Massachusetts, United States

2 Department of Pediatrics, Harvard Medical School, Boston, Massachusetts, United States

3 Program in Cellular and Molecular Medicine, Boston Children's Hospital, Boston, Massachusetts, United States

${ }^{4}$ Department of Medicine, Harvard Medical School, Boston,

Massachusetts, United States

${ }^{5}$ Division of Gastroenterology, Brigham and Women's Hospital, Boston, Massachusetts, United States

${ }^{6}$ Division of Hematology/Oncology, Boston Children's Hospital, Boston, Massachusetts, United States
}

\author{
Address for correspondence Denisa D. Wagner, PhD, Program in \\ Cellular and Molecular Medicine, Boston Children's Hospital, \\ 3 Blackfan Circle, Third Floor, Boston, MA 02115 United States \\ (e-mail: Denisa.Wagner@childrens.harvard.edu).
}

TH Open 2017;1:e11-e23.

\begin{abstract}
Keywords

- ADAMTS13

- colitis

- Crohn's disease

- thrombosis

- VWF

Background Inflammatory bowel disease (IBD) affects 1.6 million people in the United States. IBD is associated with an increased risk of thrombosis, which rises with disease activity. The pathogenesis of IBD and its increased thrombotic risk is not completely understood. Ultra large von Willebrand factor (ULVWF) multimers are secreted from activated endothelium, leading to recruitment of platelets and leukocytes. A disintegrin and metalloproteinase with thrombospondin type I repeats motif 13 (ADAMTS13) cleaves highly adhesive ULVWF into smaller, less bioactive, multimers, releasing them into circulation. Mice deficient in ADAMTS13 (ADAMTS13 ${ }^{-l-}$ ) have heightened inflammatory and thrombotic responses.

Objectives We hypothesized that upon colitis induction, ADAMTS13 ${ }^{-I-}$ mice would have more severe symptoms compared with wild-type (WT) mice, and rhADAMTS13 administration to mice with colitis would improve their condition.

Results Dextran sodium sulfate-induced colitis was worse in ADAMTS13 ${ }^{-l-}$ mice than WT. ADAMTS13 ${ }^{-l-}$ showed increased weight loss, worse anemia, and increased clinical and histologic colitis severity, compared with WT mice. ADAMTS $13^{-1-}$ mice had increased VWF release, with accumulation at inflamed colonic sites. Also, the majority of mice showed one or more submucosal colonic thrombi. ADAMTS13 deficiency worsened colitis and propagated intestinal inflammation, most likely through increased platelet-leukocyte recruitment by VWF. Treatment of WT mice with rhADAMTS13 decreased colitis severity without worsening anemia. Additionally, several immune-mediated chronic murine colitis models, and inflamed colon tissue specimens from IBD patients, showed increased VWF release at inflamed sites, suggesting a generalizability of our findings.
\end{abstract}

DOI https://doi.org/ 10.1055/s-0037-1603927. ISSN 2512-9465.
(C) 2017 Georg Thieme Verlag KG Stuttgart · New York 
Conclusion Measuring VWF/ADAMTS13 levels could have clinical utility. When applicable, the administration of ADAMTS13, in addition to primary treatment, may improve outcomes for IBD patients.

\section{Introduction}

Inflammatory bowel disease (IBD), which includes Crohn's disease and ulcerative colitis (UC), are diseases of chronic intestinal inflammation, manifesting in relapsing and remitting (often bloody) diarrhea, as well as debilitating abdominal pain. IBD affects 1.6 million individuals in the United States ${ }^{1}$ and is rising in incidence. The precise pathogenesis of IBD is unknown; it is thought to arise from a combination of genetic predisposition and a dysregulated inflammatory response to an environmental, likely microbial, trigger. ${ }^{2}$

Interestingly, patients with IBD have a 3-fold increased risk of developing thromboembolism compared with agematched controls, and this risk rises 15 -fold with worsening disease activity ${ }^{3}$ (reviewed in Zitomersky et $\mathrm{al}^{4}$ ). Deep vein thrombosis (DVT) and pulmonary embolism (PE) are the most common thrombotic events, but arterial events, ${ }^{5}$ including cardiovascular and mesenteric ischemia, are also more prevalent in people with IBD. $^{6}$ Some postulate that thrombosis may even play a role in IBD pathogenesis. ${ }^{7-9}$ Increasing evidence links inflammation and thrombosis in multiple chronic inflammatory states, including IBD (reviewed in Esmon ${ }^{10}$ and Feys et $\mathrm{al}^{11}$ ).

Leukocyte rolling, adhesion, and transmigration are hallmarks of inflammation. Adhesion molecules on leukocytes and endothelial cells mediate this process. In fact, antibody blockade of adhesion receptors involved in trafficking of leukocytes to the gut, such as integrins, are used to treat IBD. ${ }^{12}$ The adhesion molecule, von Willebrand Factor (VWF), is a large multimeric glycoprotein. In its ultra-large form (ULVWF), VWF is stored in the Weibel-Palade bodies of the endothelium, as well as in $\alpha$-granules of platelets. ULVWF is released from endothelium upon activation by inflammatory signals or with hypoxia. ${ }^{13}$ VWF release leads to the initial adhesion of platelets and leukocytes to the vessel wall, a first step in initiating both inflammation and thrombosis. ${ }^{13,14}$ Leukocytes adhere to VWF, or to platelets recruited by the released VWF, directly through P-selectin glycoprotein ligand and $\beta 2$-integrins. ${ }^{15}$ ULVWF multimers are extremely biologically active, as they form stronger bonds with platelet GPIb. ${ }^{16}$ A disintegrin and metalloproteinase with thrombospondin type I repeats motif 13 (ADAMTS13) decreases platelet and leukocyte adhesion, as well as VWF-platelet string formation, by specifically cleaving hyperactive ULVWF multimers under conditions of fluid shear stress. ${ }^{17}$ Mice that are completely deficient in ADAMTS13 (ADAMTS13 ${ }^{-1-}$ ) have a proinflammatory and prothrombotic phenotype. ${ }^{18}$ Mice that express ADAMTS13, which contains a human mutation responsible for reducing enzymatic activity, also have a prothrombotic phenotype, ${ }^{19}$ indicating that even reduction in ADAMTS13 activity ${ }^{20}$ is deleterious. Additionally, diseases of chronic inflammation associated with thrombosis have higher plasma VWF, and reduced ADAMTS13 levels and activity, ${ }^{11}$ which could exacerbate a prothrombotic or proinflammatory state. Thus, in addition to being antithrombotic, ADAMTS13 is increasingly recognized as an anti-inflammatory mediator.

Circulating VWF is elevated in IBD patients ${ }^{11,21}$ and rises with worsening colitis disease activity, ${ }^{22}$ which suggests increased endothelial activation. In plasma from IBD subjects, ADAMTS13 levels and activity are reduced, when compared with healthy control plasma. ${ }^{11}$ Therefore, ADAMTS13 deficiency, or acquired antibody-mediated ADAMTS13 inhibition, could contribute to disease severity and increased incidence of thrombosis in this population. Several cases of patients suffering from both thrombotic thrombocytopenic purpura (thrombotic microangiopathy due to very low ADAMTS13 activity) and colitis were reported. $^{23}$

Mechanisms of platelet recruitment in colitis are not completely understood. In dextran sodium sulfate (DSS) colitis, platelets and leukocytes accumulate in venules of the inflamed colon. ${ }^{24}$ They bind endothelium and leukocytes on to the vessel wall. The presence of these adherent platelets and leukocytes in colonic vessels correlates with disease severity. ${ }^{24}$ ULVWF binds platelets and recruits leukocytes in thrombosis and inflammation, but its impact has not been extensively studied in colitis.

We evaluated the impact of ADAMTS13 deficiency on colitis induced by a chemical (DSS) in drinking water. We compared disease severity of DSS colitis in wild-type (WT) mice that were given either ADAMTS13 or a vehicle buffer. Our results indicate that ADAMTS13 deficiency leads to the accumulation of VWF-rich thrombi in colonic submucosal vessels, increasing inflamed colonic tissue, and a worse colitis phenotype. Moreover, treatment of WT mice with recombinant human ADAMTS13 (rhADAMTS13) ameliorates DSS colitis. Thus, ADAMTS13 is an important modifier in the disease.

\section{Materials and Methods}

\section{Animals}

ADAMTS13 $^{-1-} 25$ and WT mice were on a C57BL/6J background. All animals were 6- to 8-week-old males, weightmatched, in-house bred, with the majority derived from heterozygous by heterozygous crosses, and the remaining from in-house bred WT C57BL/6J. As the two genotypes compared were siblings raised in the same cage, it can be assumed that their gut microbiota were highly similar. $\mathrm{Rag}^{-/-}$, Rag2 ${ }^{-/-} \mathrm{Il}_{10 \mathrm{rb}^{-/-}}$, Il10rb ${ }^{-/-}, \mathrm{Was}^{-/-}$, and $\mathrm{Was}^{-/}$ ${ }^{-}$Il4 ${ }^{-/-}$mice were on 129 SvEv background. ${ }^{26,27}$ All experimental procedures were reviewed and approved by the Institutional Animal Care and Use Committee of Boston Children's Hospital. 


\section{Dextran Sodium Sulfate Colitis Model}

Acute DSS colitis was induced by administration of DSS salt (3\% wt/vol, mol wt: $36-50 \mathrm{kDa}$; MP Biomedicals) in autoclaved drinking water for 10 days as described. ${ }^{28}$ Fresh DSS was administered on days 3 and 6. Daily weight and clinical colitis scores were measured using an established scoring system based on percentage weight loss $(<10 \%=1$, $>10 \%=2,>20 \%=3$ ), onset of diarrhea (1), bloody diarrhea (2), hunched posture (1), and rectal prolapse (1) for a maximum score of 7.28

\section{Recombinant Human ADAMTS13 Treatment}

rhADAMTS13 was provided by F. Scheiflinger and H. Rottensteiner of Baxter Bioscience. Treatment with rhADAMTS13 (or saline vehicle) by retro-orbital intravenous injections was done on days 3 to 10 at a dose of 3,460 U/kg every 24 hours, a concentration shown to decrease leukocyte recruitment in myocardial ischemia reperfusion injury. ${ }^{29}$ This concentration was chosen due to our experience with a 10-fold lower dose of this human protein only partially dismantling mouse "VWF platelets strings" observed in activated venules by intravital microscopy. A 10-fold higher dose shows no toxicity in mice (Friedrich Scheiflinger, PhD, oral communication).

\section{Analysis of Peripheral Blood}

Blood was collected via the retro-orbital sinus into EDTAcoated capillary tubes and was analyzed by a Hemavet 950FS (Drew Scientific) for complete blood counts.

Plasma VWF, IL-6, IL-10, Soluble P-selectin, and Thrombin-Antithrombin Complexes

Plasma VWF levels were quantified by enzyme-linked immunosorbent assay (ELISA) on day $10 .^{30}$ VWF levels in plasma of WT or ADAMTS13 ${ }^{-1-}$ mice were calculated and shown as fold increase over pooled plasma VWF levels of untreated WT or untreated ADAMTS13 ${ }^{-1-}$ mice, respectively. Interleukin (IL)-6 (Biolegend), IL-10 (R\&D Systems), soluble P-selectin (sPsel; R\&D Systems), and thrombin-antithrombin (TAT) complexes (Abcam) were measured by ELISA according to the manufacturer's instructions.

\section{Human Tissue Specimens}

Anonymous human colon tissue specimens were obtained from the Pediatric Inflammatory Bowel Disease Biospecimen Repository at Boston Children's Hospital. This repository was approved by the Institutional Review Board of Boston Children's Hospital (protocol number: IRB-P00000529). Prior to specimen deposit, informed written consent was obtained from study subjects and/or their legal guardians.

\section{Tissue Preparation and Analysis}

Mice were anesthetized with isoflurane and terminally bled from the retro-orbital sinus. Entire spleens were harvested and weighed. Colon length was measured from anal verge to cecum. Then, colons were cut longitudinally and Swiss rolled so that the entire colon could be evaluated. Colons were fixed in zinc fixative (100 mM Tris- $\mathrm{HCl}$ containing $37 \mathrm{mM}$ zinc chloride, $23 \mathrm{mM}$ zinc acetate, and $3.2 \mathrm{mM}$ calcium acetate).
Paraffin-embedded sections were stained with hematoxylin and eosin (H\&E) and scored for colitis severity using an established scoring system ${ }^{31}$ by an individual blinded to the study groups. Human colon tissue biopsies were embedded in optimal cutting temperature, snap frozen, cryostat sectioned, and fixed in zinc, as described earlier.

\section{Immunostaining and Fluorescence Microscopy}

Tissue sections were washed with phosphate-buffered saline (PBS) and permeabilized (0.1\% Triton X-100, 0.1\% sodium citrate) for 10 minutes at $4^{\circ} \mathrm{C}$. Samples were blocked with $3 \%$ (wt/vol) bovine serum albumin (BSA) for 90 minutes at $37^{\circ} \mathrm{C}$, rinsed, and incubated overnight at $4^{\circ} \mathrm{C}$ or for 1 hour at $37^{\circ} \mathrm{C}$ in primary antibody dilution buffer containing $0.3 \% \mathrm{BSA}$, rabbit antihuman VWF (DAKO, 1:500), and rat antimouse CD31 (1:250, Biolegend). After several washes, samples were incubated for 2 hours at room temperature in antibody dilution buffer containing Alexa Fluor-conjugated secondary antibodies in $0.3 \%$ BSA in PBS: goat anti-rat immunoglobulin $\mathrm{G}$ (IgG) (Alexa555, $2 \mu \mathrm{g} / \mathrm{mL}$; Biolegend), donkey anti-rabbit $\operatorname{IgG}$ (Alexa488, $1.5 \mu \mathrm{g} / \mathrm{mL}$; Biolegend), or donkey anti-sheep IgG (Alexa568, $2 \mu \mathrm{g} / \mathrm{mL}$; Invitrogen). DNA was counterstained with $1 \mu \mathrm{g} / \mathrm{mL}$ Hoechst 33342 and slides were coverslipped with Fluoromount gel (Electron Microscopy Sciences). Fluorescent images were acquired using an Axiovert 200 wide field fluorescence microscope (Zeiss) in conjunction with an Axiocam MRm monochromatic CCD camera (Zeiss) and analyzed with Zeiss Axiovision software. All channels were acquired in grayscale and pseudo colored using Zeiss Axiovision or ImageJ software (National Institutes of Health). VWF immunofluorescent intensity in colon tissue was quantified by FITC intensity at the same exposure time on the same day divided by DNA area, which stained all nuclei; therefore, it estimated entire tissue area in five separate $20 \times$ images.

\section{Statistics}

Data are presented as mean \pm standard error of mean and analyzed using Student's $t$-test or Mann-Whitney $U$-test with median $\pm \mathrm{IQR}$ unless otherwise noted. Data were considered significant when $p$-values were less than 0.05 . For comparison of platelet count and weight loss after DSS colitis induction between WT and ADAMST13 ${ }^{-1-}$ mice (-Fig. 1B), an analysis of covariance was used with a 95\% confidence interval to determine if there was a difference between the two slopes.

\section{Results}

\section{DSS-Induced Colitis Is More Severe in ADAMTS13- Deficient Mice than in WT}

To address the role of ADAMTS13 in colonic inflammation, we chemically induced colitis in littermate ADAMTS13 ${ }^{-1-}$ and WT mice. Initial body weight did not differ between ADAMTS13 ${ }^{-1-}$ and WT mice, nor did either genotype have diarrhea at baseline. In three separate experiments,

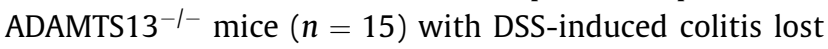
more weight compared with WT $(n=17)$ on days 9 


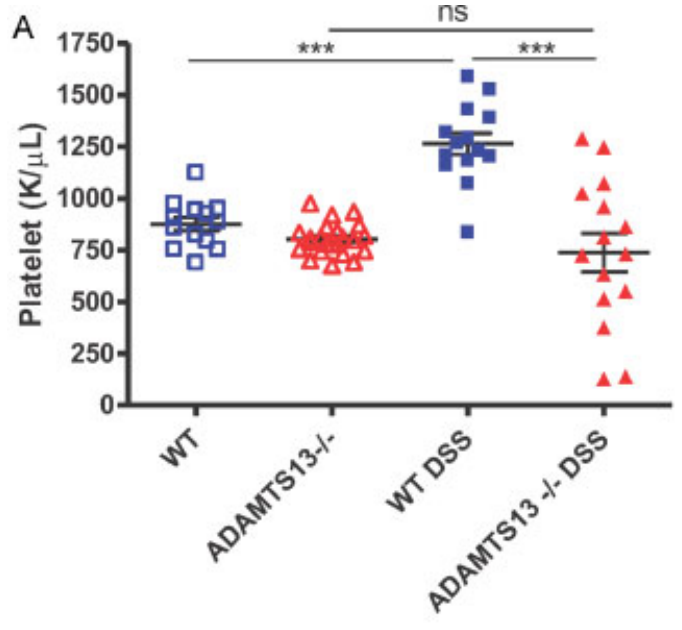

C

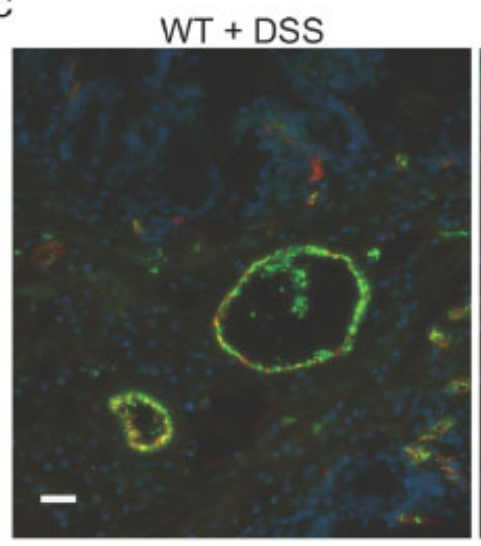

ADAMTS13-/- + DSS

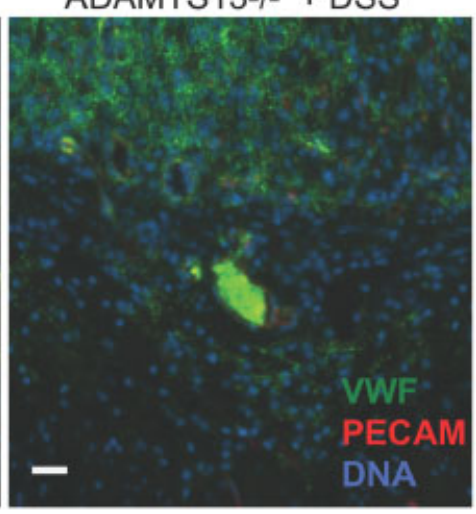

B

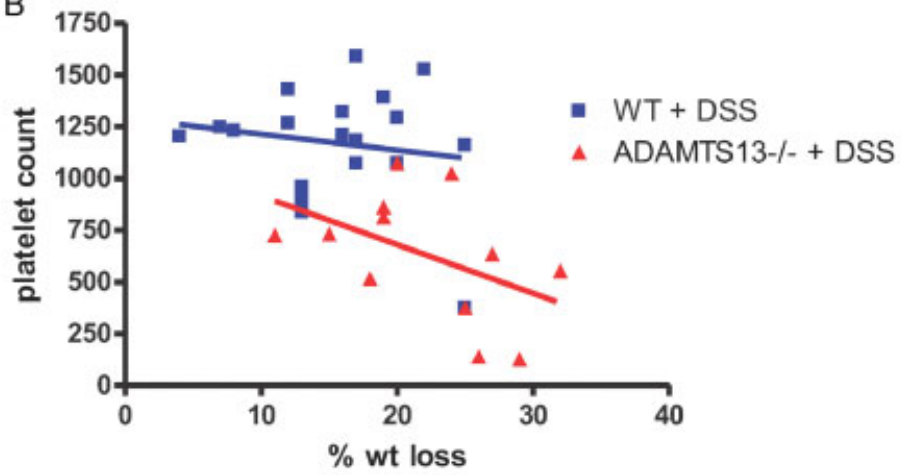

Fig. 1 Effects of ADAMTS13 deficiency on platelet count and colonic thrombosis. (A) Platelet counts for WT and ADAMTS13-/- mice without colitis did not differ. With colitis, there was significant thrombocytosis in WT mice, while in ADAMTS $13^{-1-}$ mice platelet count varied more widely but had a similar mean to baseline. (B) Platelet counts trended to be lower in mice with more weight loss, an indicator of worse colitis, and tended to decrease more in ADAMTS13 $3^{-I}$ mice with colitis (slopes $p=0.12$ ). (C) Colons from WT and ADAMTS13 ${ }^{-1-}$ mice were fluorescently stained for VWF (green), PECAM-1 (red), and DNA (blue). In WT mice, we observed increased VWF expression mostly within the vessel wall with likely some VWF-positive platelets in the lumen. In contrast, in most ADAMTS13 ${ }^{-1-}$ mice, we found a completely occlusive VWF-rich thrombus in a submucosal vessel. Entire Swiss-rolled sections of colon were inspected for thrombi. We found one or more VWF-rich thrombi in most ADAMTS13 $3^{-1-}$ colon sections and none in WT colons with colitis. In (A), mean and SEM are shown. In (C), median \pm IQR values are presented. ${ }^{*} p<0.05,{ }^{* *} p<0.005,{ }^{* * *} p<0.0005$.

Fig. 2 ADAMTS13 deficiency aggravates DSS colitis in mice. (A) Percent of original body weight in WT and ADAMTS13 $3^{-1-}$ mice. ADAMTS13 ${ }^{-1-}$ mice had significantly more weight loss on days 9 to 10 when their disease was most severe. (B) ADAMTS13 ${ }^{-1-}$ mice have a more severe colitis phenotype based on clinical colitis scores on days 8 to 10. (C) Hemoglobin concentration in WT and ADAMTS13 ${ }^{-1-}$ mice without colitis is not significantly different at baseline. With colitis, both WT and ADAMTS $13^{-1-}$ mice became anemic, but the ADAMTS $13^{-I-}$ mice became more anemic suggesting more severe disease. (D) H\&E-stained colon sections from WT and ADAMTS13 ${ }^{-1-}$ mice without colitis show normal crypt architecture with neatly aligned crypts (stars) whose bases are adjacent to the muscularis mucosa (black arrows), abundant clear mucous-filled goblet cells, preserved epithelial barrier with epithelial cells and their basolateral nuclei (diamonds), and no mucosal, submucosal, or muscularis mucosa (M) edema. Representative sections of WT mouse colon sections after DSS colitis for 10 days showing distortion of crypt architecture (branching of the normally test tube-shaped crypts), loss of goblet cells previously seen within crypts, and increased leukocytes infiltrating the lamina propria between the crypts (white starbursts). There are also more leukocytes and edema in the submucosa, which widens the submucosal space (double-headed arrow). An intact epithelial barrier remained protecting the gut from luminal contents (L). On the right panel, with increased disease severity, there was complete loss of crypt architecture and increased leukocyte infiltration, but the epithelial barrier was maintained in WT. Representative sections of ADAMTS $13^{-1-}$ colon sections show similar features as wild type but had more severe inflammation with increased crypt distortion in the middle panel and then complete loss of crypt architecture, increased leukocyte infiltration, a more widely expanded submucosal space, and transmural inflammation and ulceration with a ragged disorganized epithelial barrier. Bar $=50 \mu \mathrm{m}$. (E) Colon length was slightly shorter in ADAMTS13 ${ }^{-1-}$ mice than in WT at baseline. With colitis, ADAMTS13 ${ }^{-1-}$ mice had shorter colons than WT, suggesting more edema and inflammation. (F) Spleen weight did not differ at baseline between ADAMTS13 ${ }^{-1-}$ and WT but significantly increased in both genotypes with colitis. (G) Histologic scoring of H\&E-stained colon sections from WT and ADAMTS13 $3^{-1-}$ mice showed more severe disease in ADAMTS13 ${ }^{-1-}$ mice. (H-K) Circulating total leukocytes and subsets were measured at baseline and on day 10 of colitis. (H) Leukocyte counts did not differ at baseline but decreased more in ADAMTS13 ${ }^{-1-}$ mice with colitis. (I) Neutrophils increased in WT mice with DSS colitis but not in ADAMTS13 $3^{-1-}$ mice with colitis. (J) Lymphocytes did not differ between genotypes at baseline or with colitis. (K) Monocytes increased in both genotypes with colitis. In all graphs, mean and SEM are shown. ${ }^{*} p<0.05,{ }^{* *} p<0.005,{ }^{* * *} p<0.0005$. 

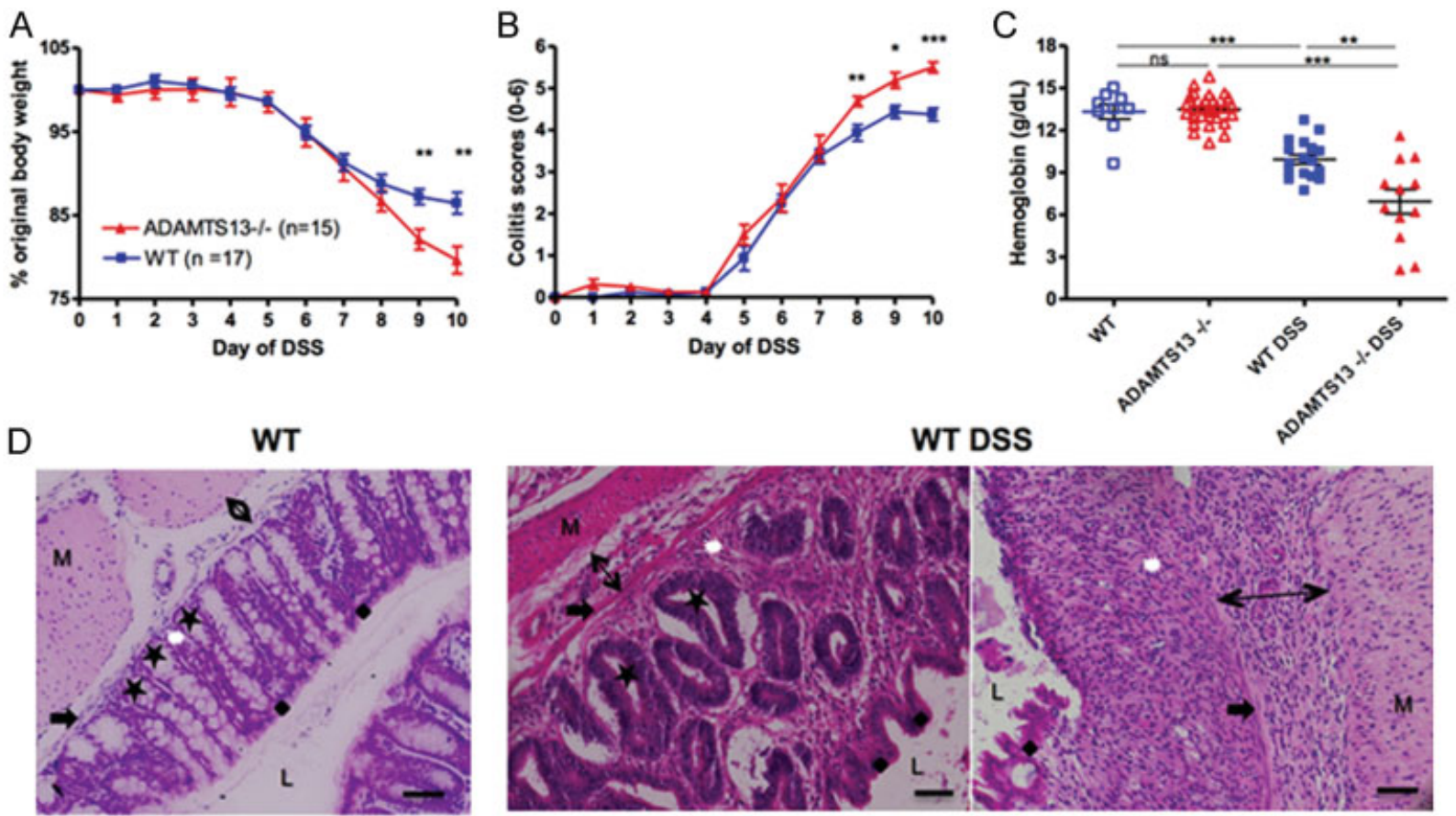

ADAMTS13-/-

ADAMTS13\%- DSS
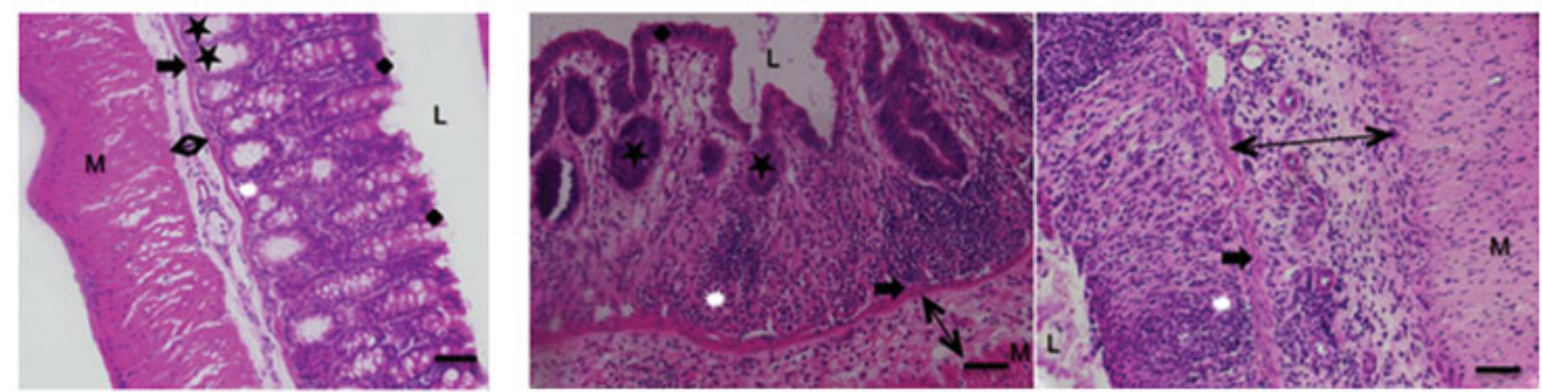

E
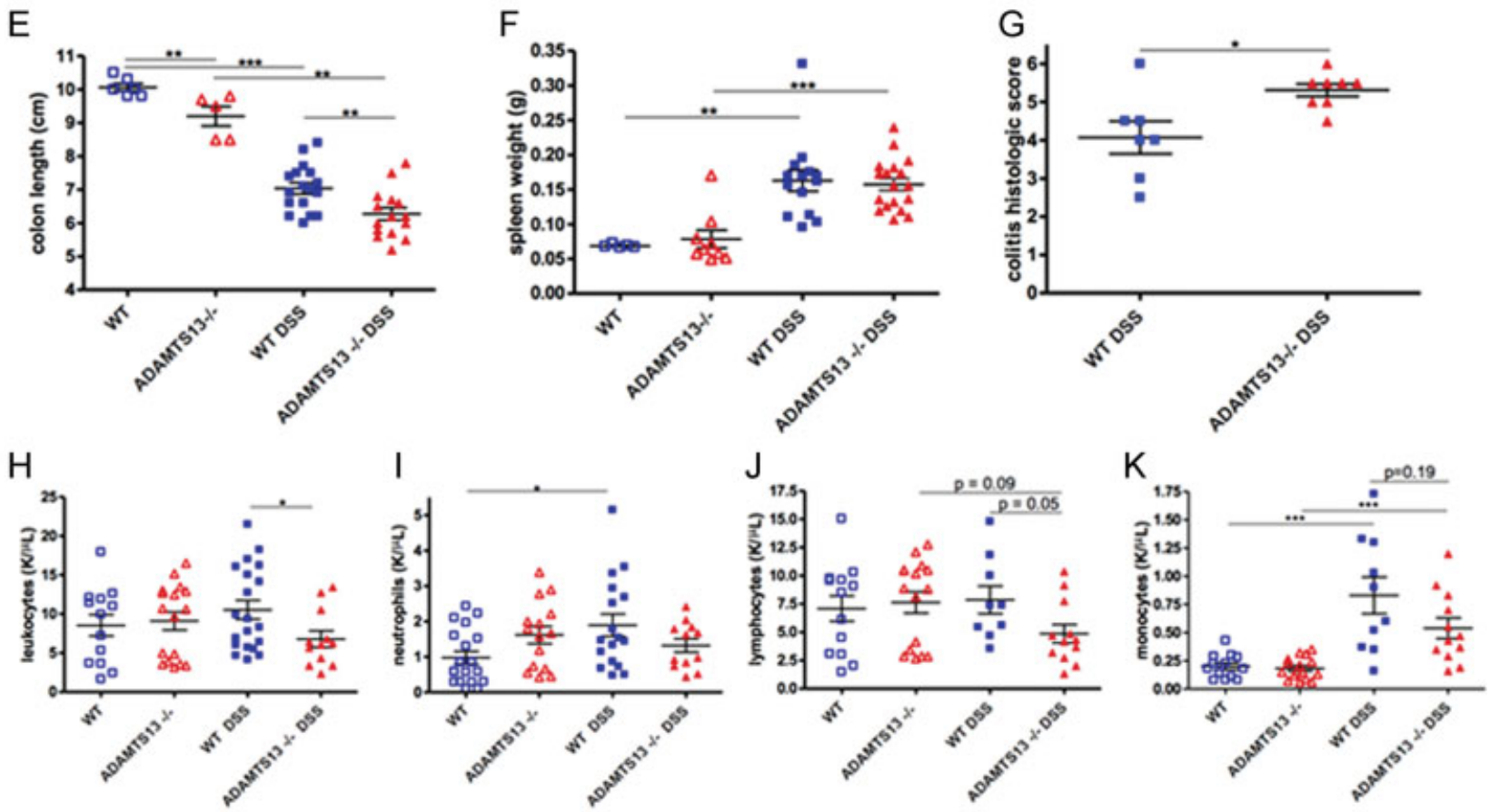

Fig. 2 (Continued) 
$(p<0.005)$ and $10(p<0.0005)$, when colitis is most severe ( - Fig. 2A). Clinical colitis scores were also higher on days 8 to 10 ( - Fig. 2B; $p<0.005, p<0.05, p<0.0005$, respectively). Higher scores in ADAMTS13 $3^{-1-}$ mice were due to more weight loss, earlier onset of bloody diarrhea, and hunched body habitus. In acute colitis, hemoglobin concentration decreases due to blood loss in stool. Hemoglobin concentration did not differ significantly at baseline prior to colitis induction, suggesting that ADAMTS13 deficiency did not result in clinically significant bleeding without intestinal mucosal injury (-Fig. 2C). ADAMTS13 $3^{-1-}$ mice developed worse anemia $(p<0.005)$ compared to WT mice with colitis (-Fig. 2C).

With colonic inflammation, the colon becomes edematous and shortens. ${ }^{28}$ ADAMTS $13^{-1-}$ mice had slightly shorter colons compared with WT mice, even without colitis ( - Fig. 2E, $p<0.005$ ). Because of this slight difference, we looked for microscopic inflammation in H\&E-stained colon sections from untreated ADAMTS13 $3^{-l-}$ mice, but we found no evidence of inflammation ( - Fig. 2D). After induction of DSS colitis, colons from ADAMTS13 $3^{-1-}$ were significantly shorter compared with WT-treated mice $(p<0.005)$ as well as untreated ADAMTS13 ${ }^{-1-}$ mice, suggesting severe colitis (-Fig. 2E). Spleen weight is a marker of systemic inflammation in colitis mouse models. Spleen weight increased significantly in both WT and ADAMTS13 $3^{-1-}$ mice with colitis $(p<0.0005)$. However, it did not differ between ADAMTS13 ${ }^{-1-}$ and WT mice (-Fig. 2F). The development of anemia, colon shortening, and increased spleen weight confirmed the induction of severe colitis in both genotypes of mice. Entire H\&E-stained Swissrolled colons were evaluated for histologic colitis severity using an established scoring system. $^{31}$ DSS-treated ADAMTS $13^{-1-}$ colons had more histologic evidence of inflammation compared with WT mice ( - Fig. $\mathbf{2 G}, p<0.05$ ) with representative sections shown in - Fig. 2D.

Blood counts revealed lower total circulating leukocytes in DSS-treated ADAMTS13 ${ }^{-1-}$ mice compared with WT mice (-Fig. 2H). Circulating leukocyte counts did not differ at baseline (-Fig. $\mathbf{2 H}$ ). Neutrophils increased in WT mice with colitis but were unchanged in ADAMTS13 $3^{-1-}$ mice with colitis (-Fig. 2I). Lymphocytes in WT mice with colitis were unchanged, with the propensity to be lower in ADAMTS13 ${ }^{-1-}$ mice, compared with WT mice (-Fig. 2J). Monocytes rose significantly in WT and ADAMTS13 $3^{-l-}$ mice with colitis, but did not differ between the two genotypes ( - Fig. 2K). No significant differences in plasma were observed in the proinflammatory cytokine IL-6, or the anti-inflammatory cytokine IL-10, TAT complexes, or sPsel between WT and ADAMTS13 ${ }^{-1-}$ mice with colitis ( - Fig. S1A-D, supplementary figure available in the online version only). These results suggest that ADAMTS13-deficiency favors the local accumulation of inflammatory cells in colonic vessels and tissue, likely without affecting systemic inflammation.

\section{ADAMTS13 ${ }^{-I-}$ Mice Drop Their Platelet Count and Develop Colonic Submucosal Thrombi with Colitis} Thrombocytosis is a marker of colonic inflammation, and is seen in colitis in mice as well as humans. ${ }^{8,32}$ We evaluated platelet counts in WT and ADAMTS13 ${ }^{-1-}$ mice before and after colitis initiation. Platelet counts in WT and ADAMTS13 ${ }^{-1-}$ mice did not differ prior to colitis initiation (-Fig. 1A). WT mice demonstrated the typical pattern of significant thrombocytosis with DSS colitis $(p<0.0005)$, whereas ADAMTS13 ${ }^{-1-}$ mice had no significant difference in platelet count after the development of colitis $(p=0.12)$. In fact, ADAMTS13 $3^{-l-}$ with the most severe colitis phenotype (indicated by more weight loss) tended to be more thrombocytopenic ( $\mathbf{- F i g}$. 1B). This finding led us to wonder whether in ADAMTS13 $3^{-1-}$ mice platelets were consumed in colonic thrombi. Therefore, we sectioned entire Swiss-rolled colons, and immunofluorescently stained them for VWF and PECAM-1 (CD31) to identify vessels. In a majority of ADAMTS13 ${ }^{-1-}$ mice with colitis, we found one or more submucosal vessel(s) completely occluded by a VWF-rich thrombus, not detected in WT ( - Fig. 1C, $p<0.05$ ). Notably, in colons from WT and ADAMTS13 ${ }^{-1-}$ mice without colitis, we found no submucosal thrombi. Taken together, these results suggest that, through accumulation of ULVWF in the colonic vessels, ADAMTS13 deficiency worsens colitis by inducing local inflammation and thrombosis.

\section{WT and ADAMTS13 ${ }^{-I-}$ Mice Show Prominent Subendothelial VWF Staining in Inflamed Colonic Tissue}

To characterize VWF in murine colitis, we quantified plasma VWF at baseline, and at day 10 of colitis. As we expected, plasma VWF increased in DSS colitis in both WT and ADAMTS $13^{-1-}$ mice. Interestingly, VWF increased significantly more in WT than in ADAMTS13 $3^{-1-}$ mice (-Fig. 3A), perhaps indicating that more ULVWF was retained in the ADAMTS $13^{-1-}$ mouse colons.

To test our hypothesis, we immunofluorescently stained colons from WT and ADAMTS13 $3^{-1-}$ mice without colitis for VWF. In healthy WT mice, VWF staining was in a dotted pattern and closely associated with PECAM-1-positive endothelium, suggesting it was still contained in endothelial Weibel-Palade bodies (-Fig. 3B). In contrast, both WT and ADAMTS13 $3^{-1-}$ mice with colitis had increased areas of VWFstained colonic tissue extending beyond PECAM-1-positive vessels into the subendothelial space (-Fig. 3B). Next, the colonic sections were assessed for VWF immunofluorescent intensity by an observer blinded to the mouse genotype and treatment. There was more intense VWF staining in colonic tissue from ADAMTS13 ${ }^{-1-}$ mice compared with WT mice with colitis $(p=0.0023$, - Fig. 3C). VWF intensity in ADAMTS $13^{-1-}$ mice increased significantly over baseline $(p=0.0012)$, while WT showed only a propensity to increase VWF tissue intensity $(p=0.07)$, indicating that ADAMTS13 is clearing the released VWF from the sites of inflammation. VWF intensity in colonic tissue did not significantly differ at baseline between WT and ADAMTS13 $3^{-l}$ mice $(p=0.43)$. This apparent retention of released VWF in inflamed tissue, along with small thrombi formation, may explain the relatively lower increase of plasma VWF in colitis in the ADAMTS13 ${ }^{-1-}$ mice compared with WT mice ( - Fig. 3A). 

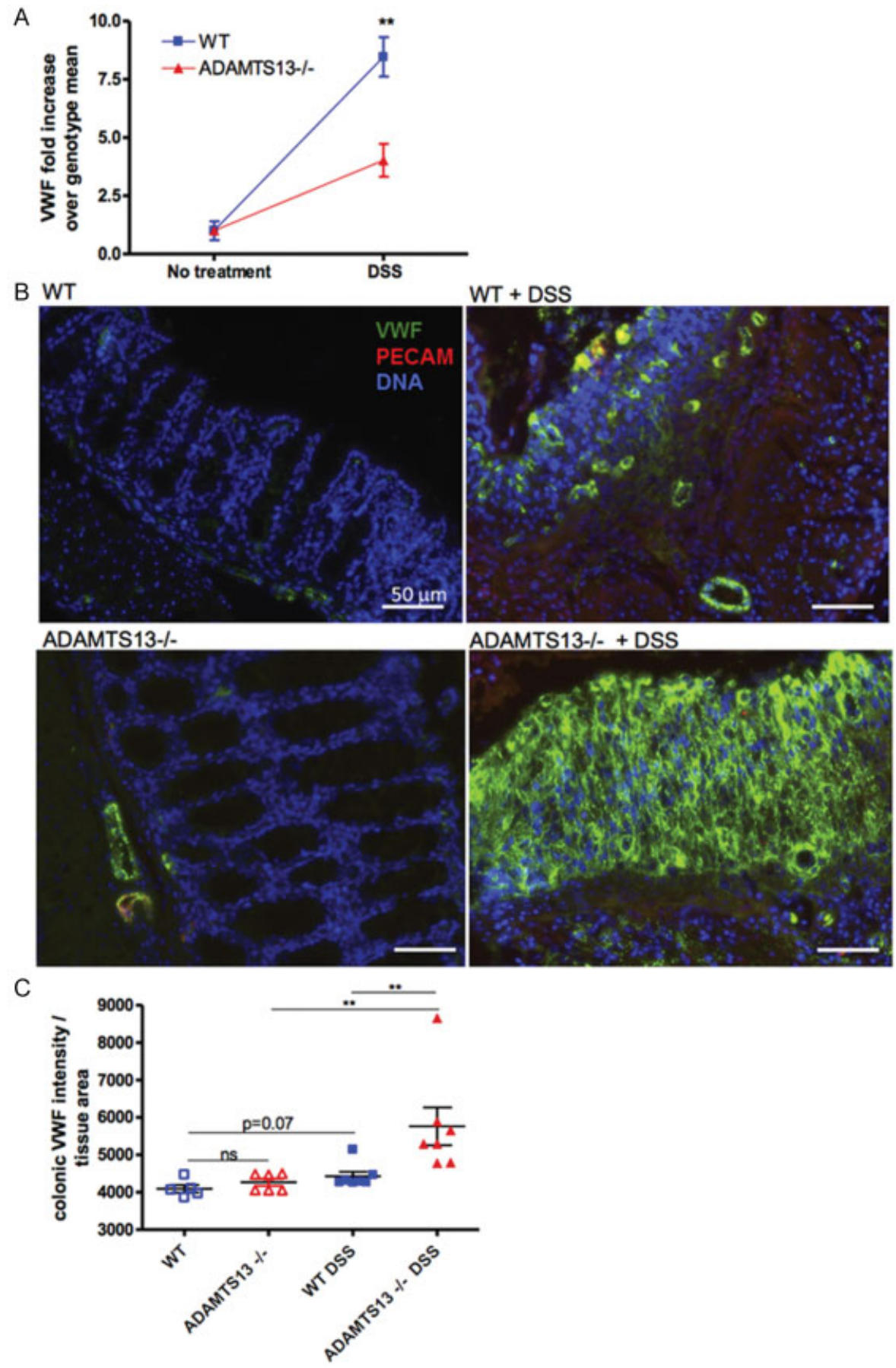

Fig. 3 Colitis causes massive VWF release. (A) WT $(n=13)$ and ADAMTS13 ${ }^{-1-}(n=9)$ mice had increased plasma VWF with colitis. The plot displays the fold increase over genotype mean without and with DSS treatment. (B) Healthy WT and ADAMTS13 mouse colons and colons from both genotypes after colitis were immunofluorescently stained for VWF, PECAM-1, and DNA. In WT mice, there was minimal VWF (green) staining seen only within vessel walls. There was not significantly more VWF stain in ADAMTS13 ${ }^{-1-}$ mouse sections without colitis. With colitis we saw an increase in VWF-positive staining which was more significant in ADAMTS13-I- mice likely due to decreased clearance of VWF from tissue. (C) Using Image J software, we quantified VWF immunofluorescence intensity divided by DNA area (to represent colon tissue area captured on the image). We confirmed higher VWF intensity in ADAMTS $13^{-1-}$ mice with colitis compared with ADAMTS $13^{-1-}$ without colitis. Furthermore, ADAMTS13 $3^{-1-}$ mice with colitis had significantly more VWF staining intensity compared with WT mice with colitis. Notably, there was no difference in WT and ADAMTS13 ${ }^{-1-}$ VWF signal intensity at baseline. There was a propensity for VWF intensity to increase with colitis in WT mice $(p=0.07)$. Mean and SEM are shown. ${ }^{* *} p<0.005$.

Increased VWF Release in Colon Tissue Is Seen in Other Murine Colitis Models and in Human Colitis Specimens In the DSS colitis model, acute colonic injury leads to the development of inflammation, mainly a Th1-mediated response, ${ }^{33}$ which is immunologically distinct from chronic colitis models, and relapsing and remitting human disease. Despite these distinctions, we hypothesized that increased colonic VWF release may also occur in additional immunemediated colitis models, as well as in human colitis. Indeed, we found prominent VWF staining in colitic tissue from 


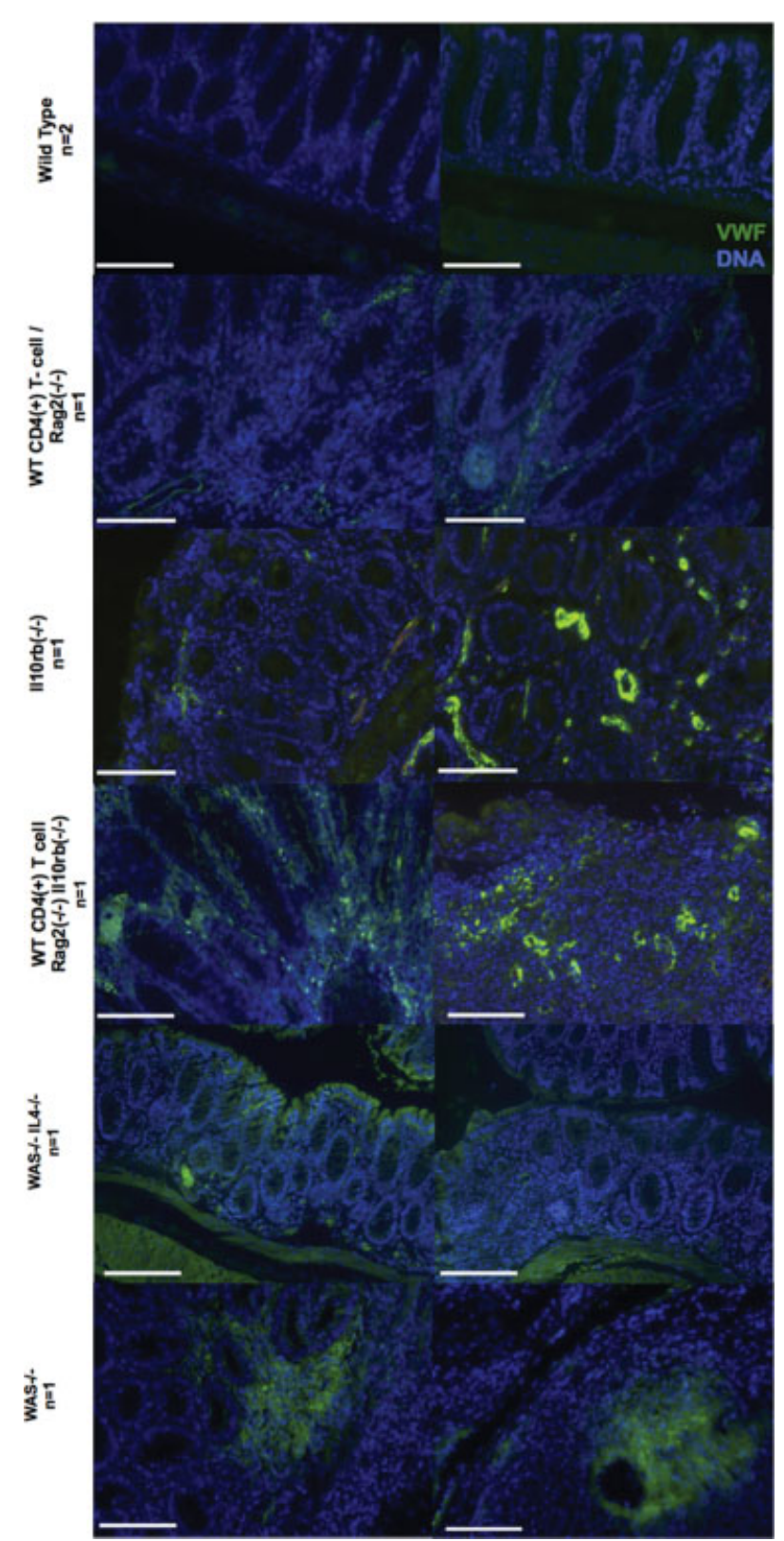

Fig. 4 Mouse models of chronic colitis show signs of VWF release in affected colonic tissue with anti-VWF fluorescent staining. WT mice colon tissue sections (top) stained for WWF (green) and DNA (blue) show minimal VWFpositive staining with faint expression within vessel walls. Colon sections from T cell transfer and chronic colitis models showed increased VWF staining dependent on the severity of the phenotype. WT CD4(+) T cell transfer into $\mathrm{Rag2}^{-/-}$and $/ 110 \mathrm{rb}^{-/-}$mice has a less severe phenotype and show less VWF staining. When WT T cells are transferred into Rag $2^{-1-} \| 10 \mathrm{rb}^{-1-}$ mice, these have a more severe phenotype and show increased VWF within and extending beyond vessels seen on the left and within neo-vessels under ulcerated tissue in the right image. Wiskott-Aldrich syndrome $\left(\mathrm{WAS}^{-1-}\right)$ protein-deficient mice develop colonic inflammation between 12 and 16 weeks which is partially driven by IL-4. A double knockout of WAS and IL4 (WAS ${ }^{-1-} \mathrm{IL} 4^{-1-}$ ) has a milder phenotype. Less WWF is seen in the milder WAS ${ }^{-1-} / \mathrm{IL}^{-1-} \mathrm{compared}^{-1}$ with the WAS $^{-1-}$. Bar $=50 \mu \mathrm{m}$.

several chronic colitis models. Interstitial VWF release seemed more pronounced in models with a more severe colitis phenotype. It is difficult to generalize among these models with various immune defects leading to colitis.
However, we can make a few comparisons. Most notably, the WT CD4 T cell transfer into Rag2 ${ }^{-1-}$ model has a mild colitis phenotype without any weight loss 4 weeks posttransfer, and minimally increased colitis scores. ${ }^{26}$ In this model, there was minimally increased colonic VWF staining compared with control WT colons and staining was contained within vessel walls (-Fig. 4). Additionally, we saw slightly increased VWF staining in the IL10 receptor $\beta$ $(\text { Il10rb })^{-1-}$ mice which have a mild phenotype with colitis development after 15 weeks. ${ }^{34}$ In contrast, when these two genotypes are combined, WT CD4 T cell transfer into Rag2 ${ }^{-1-}$ Il10 $\mathrm{rb}^{-1-}$ mice has $20 \%$ weight loss 4 weeks posttransfer and colitis scores that are six times worse than the WT CD4 T cell transfer into Rag2 $2^{-1-} .{ }^{26}$ Colons from these more severely affected mice showed extensive and intense VWF staining (-Fig. 4). Mice deficient in Wiskott Aldrich Syndrome (WAS) protein develop severe colonic inflammation by the age of 12 to 16 weeks with mixed Th1- and Th2-type responses. ${ }^{27}$ Disease in these mice is partially driven by IL4 as deletion of IL4 improves disease in $\mathrm{Was}^{-1-} I \mathrm{I}^{-/-}$mice. ${ }^{27}$ Similar to disease severity, VWF staining in colonic tissues from Was $^{-1-}$ mice is more pronounced compared with $\mathrm{Was}^{-1-} \mathrm{Il}^{-/-}$mice, which corroborates our hypothesis (-Fig. 4).

In human inflamed colonic tissue, similar to DSS colitis and the chronic colitis models mentioned earlier, we observed increased VWF release at sites of marked inflammation in comparison to healthy sites in 12 human colitis biopsy or colon resection specimens from individuals with IBD (-Fig. 5). Staining was both intravascular and extravascular with VWF extending into colon tissue ( $\mathbf{- F i g . 5 A}$ ). Together, these results suggest that even without ADAMTS13-deficiency, colitis induces the release of VWF in inflamed colonic vessels and tissue, which increases with the severity of the disease.

\section{Administration of rhADAMTS13 to WT Mice with DSS Colitis Reduces Clinical Colitis Severity}

VWF accumulates at inflamed sites in colitis, as documented earlier (-Figs. 3B and 4). VWF promotes leukocyte recruitment and transmigration, ${ }^{15,35}$ thus aggravating inflammation. Since ADAMTS13 can clear released $\mathrm{VWF}^{36}$ and endogenous ADAMTS13 partially protects WT mice in colitis as compared with mice lacking ADAMTS13 (-Figs. 1 and 2), we hypothesized that systemic administration of rhADAMTS13 to supplement endogenous ADAMTS13 in WT mice may further reduce colitis severity.

We intravenously administered rhADAMTS13 or saline vehicle daily, beginning on day 3 of DSS treatment, which is 2 days before the onset of weight loss in the DSS model. In mice treated with rhADAMTS13, we found less weight loss ( - Fig. 6A), and less severe clinical colitis scores on days 8 to 10 than in vehicle-treated mice ( - Fig. 6B). While we did observe the expected drop in hemoglobin typical of colitis with bloody diarrhea in DSS-treated mice, there was not a significant difference in hemoglobin concentrations (-Fig. 6C) or onset of bloody diarrhea (-Fig. 6E) between rhADAMTS13 and vehicle-treated mice, indicating an absence of excessive rectal bleeding as a result of the rhADAMTS13 treatment. We saw the expected increase in 


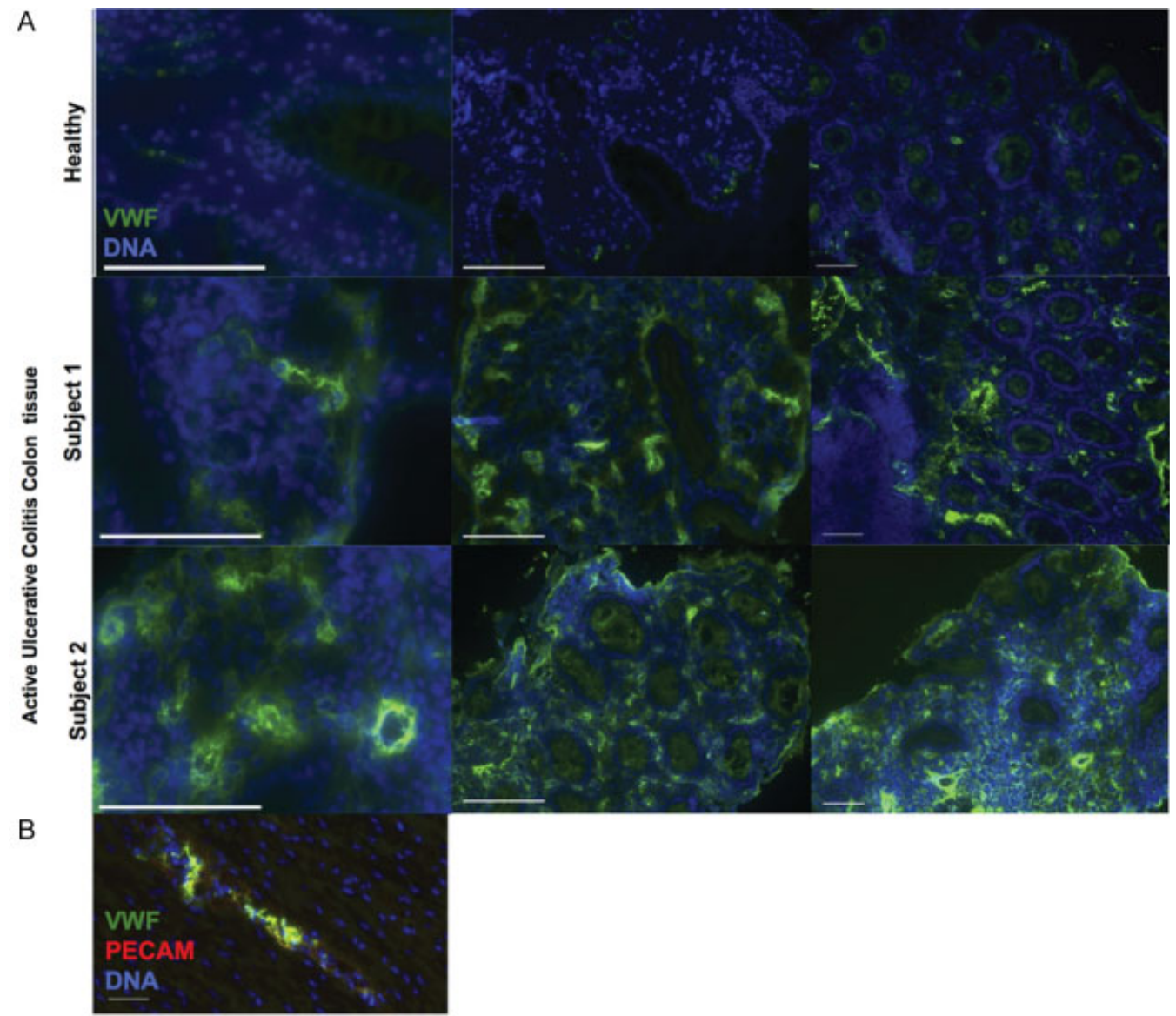

Fig. 5 Elevated VWF staining was seen in human colitis colon tissue. (A) Human colon tissue stained for VWF (green) and DNA (blue) shown at various magnifications. The top row is healthy colonic tissue with minimal VWF staining only within vessels. The second and third rows are samples from two different subjects with active ulcerative colitis (UC) and reveal increased VWF release surrounding vessels and deeper in colonic tissue. (B) A submucosal vessel (likely representing a thrombus) from a patient with UC cut longitudinally showing retained VWF ( $g r e e n$ ) inside the vessel surrounded by endothelial cells stained for PECAM-1 (red). DNA (blue) stains all cells and shows the surrounding muscularis mucosa. Bar $=50 \mu \mathrm{m}$.

platelet counts in both rhADAMTS13- and vehicle-treated groups with colitis. There tended to be less thrombocytosis in the rhADAMTS13-treated group, suggesting a possible antiinflammatory effect ( $\mathbf{- F i g . 6 D )}$. We did not find significant differences between rhADAMTS13- and vehicle-treated groups with respect to colon length, scores of microscopic inflammation of H\&E-stained colon sections, VWF colon staining, plasma VWF, or spleen weight (data not shown). This suggests that rhADAMTS13 reduced disease severity, but did not completely reverse the effect of the chemical colitis produced by DSS treatment.

No differences in IL-10, TAT, or sPsel were detected between rhADAMTS13- and vehicle-treated mice (-Fig. S1B-D, supplementary figure available in the online version only). However, administration of rhADAMTS13 resulted in lower IL-6 levels in plasma (-Fig. 6F). IL-6 is a pathologically important cytokine in human and murine colitis. ${ }^{37}$ It is both proinflammatory and prothrombotic, as it enhances platelet activation and aggregation, increases platelet leukocyte aggregates, and accelerates thrombopoiesis in DSS colitis. ${ }^{32,37}$ This finding may in part explain the beneficial effect of rhADAMT13 in the treated mice ( - Fig. 6A, B).

\section{Discussion}

Our findings show an important protective role of ADAMTS13 in colitis and provide an additional mechanism linking thrombosis and inflammation in IBD. Excessive VWF secretion from Weibel-Palade bodies has been shown to be highly proinflammatory in animal models, such as ischemia/ reperfusion injury in stroke and myocardial infarction. It appears that stress signals causing ULVWF release could also precipitate worsening of colitis by increasing inflammation and microthrombosis. Submucosal thrombi are reported in colonic biopsies from patients with IBD and not from healthy controls. ${ }^{38}$ Additionally, mesenteric venous thrombi are seen on computed tomography scans from patients with IBD and are frequently associated with areas of inflamed intestine. ${ }^{39}$ Both mesenteric vein thrombosis and mesenteric arterial thrombosis inducing ischemia are known complications of IBD. ${ }^{40}$ Our study suggests a mechanism whereby colonic inflammation releases ULVWF, which, until cleared by ADAMTS13, promotes leukocyte recruitment and entangles platelets. This results in the formation of VWF-rich thrombi in submucosal vessels. Subsequent ischemia then causes 

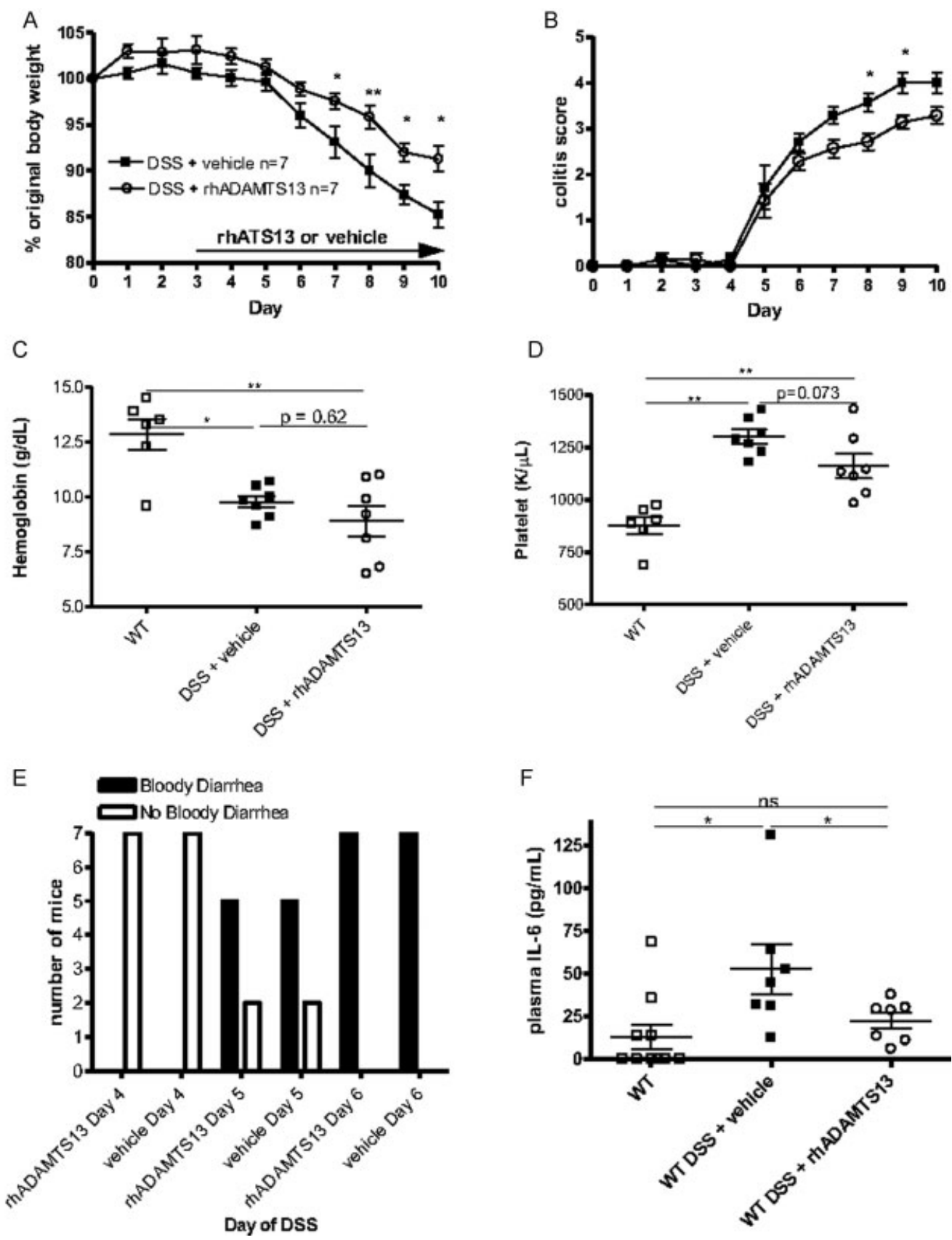

Fig. 6 Treatment of WT mice with rhADAMTS13 improves outcome. (A) Percent of original body weight in WT mice with DSS treated with vehicle (squares) or rhADAMTS13 (circles). There was significantly less weight loss in rhADAMTS13-treated mice on days 7 to 10 when colitis is most severe. (B) Colitis scores are more severe on days 8 and 9 in vehicle-treated compared with rhADAMTS13-treated mice. (C) Hemoglobin concentration decreases with colitis in both vehicle- and rhADAMTS13-treated mice. However, there is no significant difference in hemoglobin concentration between the treatment groups. (D) Platelet count increased in both groups with colitis but tended to be lower in the rhADAMTS13 treatment group. Five of seven mice in each group developed bloody diarrhea by day 5 and all mice in both groups by day 6 . (E) There was no difference in the onset of bloody diarrhea in rhADAMTS13- or vehicle-treated mice with DSS colitis. (F) Plasma IL-6, a proinflammatory and prothrombotic cytokine, was lower in rhADAMTS13-treated compared with vehicle-treated DSS colitic mice. IL-6 also increased with colitis in vehicle-treated mice but did not differ from baseline in rhADAMTS13-treated mice. In all graphs, mean and SEM are shown. ${ }^{*} p<0.05$, ${ }^{* *} p<0.005,{ }^{* * *} p<0.0005$.

more Weibel-Palade bodies to release, ${ }^{41}$ fueling the vicious circle of inflammation. Administration of pharmacological doses of ADAMTS13 to WT mice may more effectively interrupt this vicious cycle than the endogenous enzyme.

We found increased VWF release with colonic inflammation in murine DSS colitis, in several models of chronic murine colitis, and in inflamed human colon tissue samples. VWF release was most marked at ulcerated sites. Multiple factors, besides hypoxia, may contribute to endothelial activation in colitis; circulating platelet leukocyte aggregates, circulating immune complexes, infection, and cytokines (all of which could lead to Weibel-Palade body release). 
Increased vascular permeability is a feature of colitis ${ }^{24}$ and likely results from a multitude of factors, some of which are mentioned earlier. Thioglycollate peritonitis leads to increased vascular permeability of mesenteric vessels. ${ }^{35}$ In this model, antibody blockade of VWF significantly decreased vascular permeability. ${ }^{35}$ Our group has also observed that VWF release under inflammatory conditions increases the permeability of cerebral vessels. ${ }^{42}$ Thus, VWF release likely also affects colonic vessel permeability in colitis.

Excess VWF retained in colonic vessels may contribute to the poorer colitis phenotype we found in ADAMTS13 ${ }^{-1-}$ mice. We also found more VWF staining in inflamed human tissue specimens from patients with IBD compared with tissue from healthy controls ( - Fig. 5). There are minimal previous studies on VWF staining of human colon tissue, and they report varied findings. One shows an increased VWF expression in colon tissue from patients with Crohn's disease, ${ }^{43}$ while a second study found no difference in VWF staining. ${ }^{44}$ These varied findings may be a result of the heterogeneity of IBD itself, and the patchy distribution of the disease.

Our study also revealed prominent VWF staining in several chronic murine colitis models (-Fig. 4). In these models, the severity of the colitis phenotype seem to parallel the amount of VWF staining present. Similar to the human specimens, more VWF release was seen in more severely affected sites. These results suggest a generalizability of our findings, beyond the acute DSS colitis chemical injury model, to diseases of chronic intestinal inflammation, which involve both the innate and adaptive immune systems, as well as repeated immune injury, and tissue remodeling.

Our findings of increased plasma VWF in WT mice with colitis coincide with observations in human subjects, where high circulating VWF levels are found with active colitis. ${ }^{21}$ Remarkably, patients with von Willebrand disease or hemophilia have a far lower risk of developing IBD. ${ }^{45}$ This further suggests a deleterious role of VWF in colonic inflammation. VWF represents an intriguing biomarker, which could be measured in patients with IBD as an indicator of both disease activity and thrombotic risk. In this study, we have demonstrated marked VWF release in tissue from patients with active IBD ( - Fig. 5). Interestingly, one clinical study showed lower plasma ADAMTS13 activity associated with elevated CRP in patients with IBD compared with healthy controls. ${ }^{11}$ However, why ADAMTS13 activity may be lower in IBD $^{11}$ is not clear. If it is produced by a mutation or polymorphism, it could be a genetic modifier predisposing to the disease. Low activity could also be the result of the disease, as the enzyme might be consumed by excessive release of VWF and VWFmediated platelet aggregate formation. High circulating VWF and decreased ADAMTS13 activity could be used as indicators for patients who would benefit from anticoagulation and/or rhADAMTS13 to decrease their thrombosis risk with an added anti-inflammatory effect.

In our study, treatment of WT mice with rhADAMTS13 decreased colitis severity. Of note, despite active colonic bleeding in colitis, rhADAMTS13 treatment did not worsen anemia. This is an important feature if it is to be considered as an adjunct treatment in colitis.
Thrombocytosis is an inflammatory marker in colitis. ${ }^{8}$ The trend toward less thrombocytosis in rhADAMTS13-treated mice suggests an anti-inflammatory effect. Additionally, rhADAMTS13 treatment resulted in a reduction of plasma IL-6. IL-6 is a proinflammatory cytokine associated with both murine and human colitis. ${ }^{46}$ IL- 6 increases platelet production, and these newly formed platelets are more thrombogenic. ${ }^{10,47}$ Intermittent ischemia and reperfusion occur in the affected areas of the colon. rhADAMTS13 treatment improves ischemia/reperfusion injury in animal models of stroke $^{48}$ and myocardial infarction, ${ }^{29,49,50}$ both of which are VWF mediated. These examples, combined with the observed release of VWF in our study, support another potential anti-inflammatory mechanism for rhADAMTS13 in colitis along the ADAMTS13-VWF axis.

Our findings suggest that VWF and ADAMTS13 play significant roles in modulating colitis. Elevated plasma VWF and decreased ADAMTS13 levels or activity are intriguing biomarkers of inflamed endothelium and thrombotic risk in patients with IBD, which should be further investigated in human subjects. In patients with low ADAMTS13, rhADAMTS13 could provide additional anti-inflammatory and antithrombotic effects, thus improving upon existing immunomodulatory therapies used in IBD.

\section{Funding}

This work was supported by the National Heart, Lung, and Blood Institute of the National Institutes of Health grants R01HL102101, R35HL135765 (to D.D.W.), and 5T32HL066987-13 (K.M.), and by Basic Research Grant H13-20955 from Baxter Bioscience (to D.D.W.).

Conflict of Interest

None declared.

\section{Acknowledgments}

rhADAMTS13 was provided by $F$. Scheiflinger and $H$. Rottensteiner (Baxalta GmbH, Vienna, Austria). N.L.Z. was supported by the division of Gastroenterology, Hepatology and Nutrition and Pediatric Inflammatory Bowel Disease Center at Boston Children's Hospital, Boston, MA. We would like to thank Haley Larson for helping with the preparation of the manuscript.

\section{References}

1 Kappelman MD, Moore KR, Allen JK, Cook SF. Recent trends in the prevalence of Crohn's disease and ulcerative colitis in a commercially insured US population. Dig Dis Sci 2013;58(02): 519-525

2 Xavier RJ, Podolsky DK. Unravelling the pathogenesis of inflammatory bowel disease. Nature 2007;448(7152):427-434

3 Bernstein CN, Blanchard JF, Houston DS, Wajda A. The incidence of deep venous thrombosis and pulmonary embolism among 
patients with inflammatory bowel disease: a population-based cohort study. Thromb Haemost 2001;85(03):430-434

4 Zitomersky NL, Verhave M, Trenor CC III. Thrombosis and inflammatory bowel disease: a call for improved awareness and prevention. Inflamm Bowel Dis 2011;17(01):458-470

5 Ha C, Magowan S, Accortt NA, Chen J, Stone CD. Risk of arterial thrombotic events in inflammatory bowel disease. Am J Gastroenterol 2009;104(06):1445-1451

6 Ozturk K, Guler AK, Cakir M, et al. Pulse wave velocity, intima media thickness, and flow-mediated dilatation in patients with normotensive normoglycemic inflammatory bowel disease. Inflamm Bowel Dis 2015;21(06):1314-1320

7 Danese S, Papa A, Saibeni S, Repici A, Malesci A, Vecchi M. Inflammation and coagulation in inflammatory bowel disease: the clot thickens. Am J Gastroenterol 2007;102(01):174-186

8 Harries AD, Fitzsimons E, Fifield R, Dew MJ, Rhoades J. Platelet count: a simple measure of activity in Crohn's disease. Br Med J (Clin Res Ed) 1983;286(6376):1476

9 Lentz SR. Thrombosis in the setting of obesity or inflammatory bowel disease. Blood 2016;128(20):2388-2394

10 Esmon CT. The impact of the inflammatory response on coagulation. Thromb Res 2004;114(5-6):321-327

11 Feys HB, Canciani MT, Peyvandi F, Deckmyn H, Vanhoorelbeke K, Mannucci PM. ADAMTS13 activity to antigen ratio in physiological and pathological conditions associated with an increased risk of thrombosis. Br J Haematol 2007;138(04):534-540

12 Feagan BG, Rutgeerts P, Sands BE, et al; GEMINI 1 Study Group. Vedolizumab as induction and maintenance therapy for ulcerative colitis. N Engl J Med 2013;369(08):699-710

13 Wagner DD. Cell biology of von Willebrand factor. Annu Rev Cell Biol 1990;6:217-246

14 Ley K, Laudanna C, Cybulsky MI, Nourshargh S. Getting to the site of inflammation: the leukocyte adhesion cascade updated. Nat Rev Immunol 2007;7(09):678-689

15 Pendu R, Terraube V, Christophe OD, et al. P-selectin glycoprotein ligand 1 and beta2-integrins cooperate in the adhesion of leukocytes to von Willebrand factor. Blood 2006;108(12):3746-3752

16 Sporn LA, Marder VJ, Wagner DD. von Willebrand factor released from Weibel-Palade bodies binds more avidly to extracellular matrix than that secreted constitutively. Blood 1987;69(05):1531-1534

17 Dong JF, Moake JL, Nolasco L, et al. ADAMTS-13 rapidly cleaves newly secreted ultralarge von Willebrand factor multimers on the endothelial surface under flowing conditions. Blood 2002;100 (12):4033-4039

18 Chauhan AK, Kisucka J, Brill A, Walsh MT, Scheiflinger F, Wagner DD. ADAMTS13: a new link between thrombosis and inflammation. J Exp Med 2008;205(09):2065-2074

19 De Cock E, Hermans C, De Raeymaecker J, et al. The novel ADAMTS13-p.D187H mutation impairs ADAMTS13 activity and secretion and contributes to thrombotic thrombocytopenic purpura in mice. J Thromb Haemost 2015;13(02):283-292

20 Banno F, Chauhan AK, Kokame K, et al. The distal carboxylterminal domains of ADAMTS13 are required for regulation of in vivo thrombus formation. Blood 2009;113(21):5323-5329

21 Stevens TR, James JP, Simmonds NJ, et al. Circulating von Willebrand factor in inflammatory bowel disease. Gut 1992;33(04): 502-506

22 Zezos P, Papaioannou G, Nikolaidis N, Vasiliadis T, Giouleme O, Evgenidis N. Elevated plasma von Willebrand factor levels in patients with active ulcerative colitis reflect endothelial perturbation due to systemic inflammation. World J Gastroenterol 2005;11(48):7639-7645

23 Ahmed S, Siddiqui AK, Chandrasekaran V. Correlation of thrombotic thrombocytopenic purpura disease activity with von Willebrand factor-cleaving protease level in ulcerative colitis. Am J Med 2004;116(11):786-787

24 Mori M, Salter JW, Vowinkel T, Krieglstein CF, Stokes KY, Granger DN. Molecular determinants of the prothrombogenic phenotype assumed by inflamed colonic venules. Am J Physiol Gastrointest Liver Physiol 2005;288(05):G920-G926

25 Chauhan AK, Walsh MT, Zhu G, Ginsburg D, Wagner DD, Motto DG The combined roles of ADAMTS13 and VWF in murine models of TTP, endotoxemia, and thrombosis. Blood 2008;111(07):3452-3457

26 Shouval DS, Biswas A, Goettel JA, et al. Interleukin-10 receptor signaling in innate immune cells regulates mucosal immune tolerance and anti-inflammatory macrophage function. Immunity 2014;40(05):706-719

27 Nguyen DD, Maillard MH, Cotta-de-Almeida V, et al. Lymphocytedependent and Th2 cytokine-associated colitis in mice deficient in Wiskott-Aldrich syndrome protein. Gastroenterology 2007; 133(04):1188-1197

28 Wirtz S, Neurath MF. Mouse models of inflammatory bowel disease. Adv Drug Deliv Rev 2007;59(11):1073-1083

29 Savchenko AS, Borissoff JI, Martinod K, et al. VWF-mediated leukocyte recruitment with chromatin decondensation by PAD4 increases myocardial ischemia/reperfusion injury in mice. Blood 2014;123(01):141-148

30 Brill A, Fuchs TA, Chauhan AK, et al. von Willebrand factormediated platelet adhesion is critical for deep vein thrombosis in mouse models. Blood 2011;117(04):1400-1407

31 Mizoguchi E. Chitinase 3-like-1 exacerbates intestinal inflammation by enhancing bacterial adhesion and invasion in colonic epithelial cells. Gastroenterology 2006;130(02):398-411

32 Yan SL, Russell J, Harris NR, Senchenkova EY, Yildirim A, Granger DN. Platelet abnormalities during colonic inflammation. Inflamm Bowel Dis 2013;19(06):1245-1253

33 Alex P, Zachos NC, Nguyen T, et al. Distinct cytokine patterns identified from multiplex profiles of murine DSS and TNBSinduced colitis. Inflamm Bowel Dis 2009;15(03):341-352

34 Spencer SD, Di Marco F, Hooley J, et al. The orphan receptor CRF24 is an essential subunit of the interleukin 10 receptor. J Exp Med 1998;187(04):571-578

35 Petri B, Broermann A, Li H, et al. von Willebrand factor promotes leukocyte extravasation. Blood 2010;116(22):4712-4719

36 Motto DG, Chauhan AK, Zhu G, et al. Shigatoxin triggers thrombotic thrombocytopenic purpura in genetically susceptible ADAMTS13-deficient mice. J Clin Invest 2005;115(10): 2752-2761

37 Senchenkova EY, Komoto S, Russell J, et al. Interleukin-6 mediates the platelet abnormalities and thrombogenesis associated with experimental colitis. Am J Pathol 2013;183(01):173-181

38 Dhillon AP, Anthony A, Sim R, et al. Mucosal capillary thrombi in rectal biopsies. Histopathology 1992;21(02):127-133

39 Violi NV, Schoepfer AM, Fournier N, Guiu B, Bize P, Denys A; Swiss Inflammatory Bowel Disease Cohort Study Group. Prevalence and clinical importance of mesenteric venous thrombosis in the Swiss Inflammatory Bowel Disease Cohort. AJR Am J Roentgenol 2014; 203(01):62-69

40 Papay P, Miehsler W, Tilg H, et al. Clinical presentation of venous thromboembolism in inflammatory bowel disease. J Crohn's Colitis 2013;7(09):723-729

41 Pinsky DJ, Naka Y, Liao H, et al. Hypoxia-induced exocytosis of endothelial cell Weibel-Palade bodies. A mechanism for rapid neutrophil recruitment after cardiac preservation. J Clin Invest 1996;97(02):493-500

42 Suidan GL, Brill A, De Meyer SF, et al. Endothelial Von Willebrand factor promotes blood-brain barrier flexibility and provides protection from hypoxia and seizures in mice. Arterioscler Thromb Vasc Biol 2013;33(09):2112-2120

43 Middleton J, Americh L, Gayon R, et al. A comparative study of endothelial cell markers expressed in chronically inflamed human tissues: MECA-79, Duffy antigen receptor for chemokines, von Willebrand factor, CD31, CD34, CD105 and CD146. J Pathol 2005;206(03):260-268

44 MatsumotoT, Kitano A, Nakamura S, et al. Possible role of vascular endothelial cells in immune responses in colonic mucosa 
examined immunocytochemically in subjects with and without ulcerative colitis. Clin Exp Immunol 1989;78(03):424-430

45 Thompson NP, Wakefield AJ, Pounder RE. Inherited disorders of coagulation appear to protect against inflammatory bowel disease. Gastroenterology 1995;108(04):1011-1015

46 Powell N, Lo JW, Biancheri P, et al. Interleukin 6 increases production of cytokines by colonic innate lymphoid cells in mice and patients with chronic intestinal inflammation. Gastroenterology 2015;149(02):456-467

47 Burstein SA. Cytokines, platelet production and hemostasis. Platelets 1997;8(2-3):93-104
48 Khan MM, Motto DG, Lentz SR, Chauhan AK. ADAMTS13 reduces VWF-mediated acute inflammation following focal cerebral ischemia in mice. J Thromb Haemost 2012;10(08): 1665-1671

49 De Meyer SF, Savchenko AS, Haas MS, et al. Protective antiinflammatory effect of ADAMTS13 on myocardial ischemia/reperfusion injury in mice. Blood 2012;120(26):5217-5223

50 Gandhi C, Motto DG, Jensen M, Lentz SR, Chauhan AK. ADAMTS13 deficiency exacerbates VWF-dependent acute myocardial ischemia/reperfusion injury in mice. Blood 2012;120 (26):5224-5230 